\title{
A Retrospective Analysis on Risk Factors for 30- day Readmission Rates in Patients Living With HIV and Severe Major Depression Disorder
}

\author{
Sindhura Kompella ${ }^{1}$, Joseph Ikekwere ${ }^{2}$, Clara Alvarez ${ }^{1}$, Ian H. Rutkofsky ${ }^{1}$ \\ 1. Psychiatry, Aventura Hospital and Medical Center, Aventura, USA 2. Psychiatry/Addiction, University of Illinois at \\ Chicago, Chicago, USA
}

Corresponding author: Joseph Ikekwere, ikejoseph20@gmail.com

\section{Abstract \\ Background}

Major depression disorder (MDD) is the most common psychiatric comorbidity in patients living with HIV (PLWHIV). The prevalence rate of MDD is higher in PLWHIV in comparison to the general population. In our study, we focus specifically on the 30-day readmission rate of PLWHIV and severe major depression.

\section{Methods}

The Health Care Agency (HCA) databank was used to conduct a retrospective study on PLWHIV and severe MDD. Keywords such as HIV, severe MDD, CD4, viral load were used to identify the data. 30-day readmission rate is studied in PLWHIV and severe MDD (N=143). Variables such as age, sex, gender, adherence to antiretroviral medications, cluster of differentiation 4 (CD4), and viral load were studied in this population. Diagnostic and Statistical Manual of Mental Disorders, 5th Edition (DSM-5) criteria were used to diagnose severe MDD in PLWHIV. An antiretroviral therapy (ART) medication list was used to analyze adherence in this population group. Geographical locations were identified using urbanization codes.

\section{Results}

Logistic regression analysis for the 30-day readmission rate in PLWHIV was found to be higher in the older age group $(\mathrm{p}<0.01)$. Caucasian population $(\mathrm{p}<0.01)$ and rural areas $(\mathrm{p}<0.01)$, ART non-adherence $(\mathrm{p}<0.05)$, and severe major depression were also found to be significant in this population $(\mathrm{p}<0.01)$.

\section{Conclusion}

As more patients live longer with HIV/AIDS, it gives rise to illnesses such as anxiety, depression, and cognitive impairment. Thus, it is important to identify severe depression in PLWHIV since it can have an impact on rates of hospitalization, morbidity/mortality, and the financial burden, specifically within 30days of discharge.

Review began 06/01/2021 Review ended 06/16/2021 Published 06/24/2021

(๑) Copyright 2021

Kompella et al. This is an open access article distributed under the terms of the Creative Commons Attribution License CC-BY 4.0., which permits unrestricted use, distribution, and reproduction in any medium, provided the original author and source are credited.
Categories: Psychiatry, HIV/AIDS, Epidemiology/Public Health

Keywords: hiv, minority, hospital readmission rate, major depressive disorder, people living with hiv/aids

\section{Introduction}

The 30-day readmission rates have become an integral quality care metric across healthcare since they may be associated with an increase in financial costs, psychosocial stressors, and mortality [1]. People living with HIV (PLWHIV) have been shown to have higher rates of readmission compared to the general population, despite the advances in HIV treatment in the form of highly active antiretroviral therapy (HAART) [2].

The earlier years of the Acquired Immunodeficiency Syndrome (AIDS) epidemic resulted in hospitalizations and readmissions based on the novelty of the disease pathology and lack of efficacious treatment modalities. While the HAART era in the 1990s ushered in a period of steady decline associated with AIDS-related complications, it also resulted in an increase in the rates of hospitalizations and readmissions due to an increase in mental healthcare burden in this population group [3].

While studies have been conducted on readmission rates in PLWHIV focusing on preventative measures and management techniques, they have been found too broad in their scope of review [3]. Moreover, these studies are lacking in delineating which specific risk factors are associated with mental illnesses in HIV or 30-day readmission rates in this population group.

Since depression is the most prevalent mental health disorder in PLWHIV, this study aims to study severe major depression disorder (MDD) in PLWHIV [3], while outlining the contributing risk factors for 30-day 


\section{Cureus}

readmission rates in this population group with co-occurring severe MDD [4].

\section{Materials And Methods}

This study was conducted in accordance with the Declaration of Helsinki and appropriate Institutional Review Board (IRB) approval was obtained. A waiver of informed consent was deemed appropriate by the IRB. We utilized the Health Care Agency (HCA) enterprise-level database to retrospectively study (20152020) the impact of depression on PLWHIV. Inclusion criteria included demographics such as gender: male or female; age: 18-85 years, and ethnicity: Caucasian, African American, Hispanic, and Other. Only inpatient hospital discharges and readmission data is obtained using the keywords HIV, MDD, CD4 count, and viral load.

Each patient's viral load was evaluated as a binary outcome with detectable viral load copies ranging from $>20$ copies/ml to undetectable. CD4 count $<500$ was used as an inclusionary criterion for determining HIV. CD4 count $<200$ was determined as AIDS. The antiretroviral therapy (ART) medications analyzed comprised any available single-dose, combination medication, or ART cocktail comprising of three or more medications including integrase strand transfer inhibitors (INSTIs), nucleoside/nucleotide reverse transcriptase inhibitors (NRTIs), non-nucleoside reverse transcriptase inhibitors (NNRTIs), cytochrome P4503A (CYP3A) inhibitors, protease Inhibitors (PIs), fusion inhibitors (FIs), post-attachment inhibitors, chemokine coreceptor antagonists (CCR5 antagonists), and entry inhibitors.

Diagnostic and Statistical Manual of Mental Disorders, 5th Edition (DSM-5) criteria were used: F33.2 [5], while ICD 10 codes: F33.0, F33.1, F33.3, F33.4, F33.9 (mild, moderate severity, psychotic features, etc) were excluded. The index hospitalizations determined were day 1 and day 30 and included hospitalizations for patients being treated for HIV/AIDS. Urbanization codes were established utilizing the Environmental Systems Research Institute (ESRI) demographics tapestry segmentation system and these codes were used to distinguish urban and rural geographical localities into further sub-geographic locations consisting of metro cities, principal urban centers, rural, semirural, suburban periphery, and urban periphery [6]. Insurance status was also reviewed as private, government, or no insurance. The insurance variable was used as a measure of socioeconomic status. The data was analyzed using binary logistic regression analysis to predict 30-day readmission rates in PLWHIV and severe MDD.

\section{Results}

In our study, there were a total of $\mathrm{n}=143$ PLWHIV and severe MDD identified who were readmitted within 30 days of discharge. The mean age for this population group was found to be 60 years (Table 1). Increasing age is found to be a significant risk factor for readmission within 30 days and the Caucasian population is more likely to be readmitted within 30 days compared to other ethnicities $(\mathrm{p}<0.001)$.

\begin{tabular}{|c|c|c|c|}
\hline Demographics & Sig value $(p)$ & 95\% Cl for EXP Lower & $95 \% \mathrm{Cl}$ for EXP Upper \\
\hline Age & 0.002 & 0.987 & 0.997 \\
\hline Gender & 0.730 & 0.708 & 6.079 \\
\hline Race (Caucasian) & 0.001 & 0.473 & 0.616 \\
\hline ART non-adherence & 0.005 & 0.190 & 0.593 \\
\hline
\end{tabular}

TABLE 1: Logistic regression for 30-day readmission rate in PLWHIV, severe MDD: demographics: age, gender, and race; ART adherence.

ART, antiretroviral therapy; EXP, exponential; sig, significant; PLHIV, people living with HIV; MDD, major depression disorder

Controlling for the other variables in the logistic regression analysis, we also found that patients with ART non-adherence were more likely to be readmitted within 30 days of discharge when compared to patients with adherence to ART $(\mathrm{p}<0.05)$.

Additionally, uninsured patients and government insurance were more likely to be readmitted in a 30-day interval compared to those with private insurance $(\mathrm{p}<0.0001)$ (Table 2). Also, patients living in suburban periphery areas (urbanization codes: R, SP) were also more likely to be readmitted within 30 days of discharge in comparison to urban areas (urbanization codes: MC, PUC, SR; p<0.005) (Table 3). 


\section{Cureus}

\begin{tabular}{|c|c|c|c|}
\hline Insurance status & Sig value $(p)$ & 95\% Cl for EXP Lower & 95\% Cl for EXP Upper \\
\hline Insurance (Government) & 0.000 & 1.315 & 1.661 \\
\hline No insurance & 0.000 & 1.315 & 1.861 \\
\hline Insurance (Private) & 0.817 & 0.780 & 1.217 \\
\hline
\end{tabular}

TABLE 2: Logistic regression for 30-day readmission rate in PLWHIV, severe MDD, and insurance status.

EXP, exponential; sig, significant; PLHIV, people living with HIV; MDD, major depression disorder

\begin{tabular}{|c|c|c|c|}
\hline Urbanization codes & Sig value $(p)$ & 95\% Cl for EXP Lower & 95\% Cl for EXP Upper \\
\hline Urbanization (MC) & 0.644 & 0.891 & 1.206 \\
\hline Urbanization (PUC) & 0.322 & 0.883 & 1.459 \\
\hline Urbanization (R) & 0.050 & 0.768 & 0.813 \\
\hline Urbanization (SR) & 0.239 & 0.924 & 1.372 \\
\hline Urbanization (SP) & 0.006 & 0.648 & 0.929 \\
\hline
\end{tabular}

TABLE 3: Logistic regression for 30-day readmission rate in PLWHIV, severe MDD, and urbanization codes.

Sig, significant; EXP, exponential; MC, metro cities; PUC, principal urban centers; R, rural; SR, suburban rural; SP, suburban periphery; PLHIV, people living with HIV; MDD, major depression disorder

\section{Discussion}

Pharmacological advancements and biopsychosocial approach towards HIV/AIDs treatment have contributed to longer life expectance. However, longevity in survival has increased AIDS-related medical complications and mental illnesses such as anxiety, cognitive impairment, and the most debilitating of mental health pathologies-depression [7]. Our result shows the statistical significance of the 30-day readmission rate among PLWHIV and severe depression. Additionally, below is a summary of some of the risk factors which may contribute to an increase in the 30-day readmission rate in this population group.

\section{Demographics}

Age

An increment in age showed an increase in the likelihood for readmission within 30 days in PLWHIV and severe depression. This may be since this population group presents with severe medical comorbidities as a result of the progression of the illness. Moreover, comorbid severe depression can complicate treatment compliance due to feelings of anhedonia or social withdrawal [7].

Race

Our result demonstrated that Caucasian ethnicity was more likely to be readmitted within 30 days in comparison to Black, Hispanic, or other ethnicities among PLWHIV and severe depression. Contrary to the literature review for minorities versus Caucasians, interestingly, our study found a lower likelihood of readmission among the minority groups $[8,9]$. This may be due to socio-cultural determinants of health including healthcare access barriers, health-seeking beliefs/behaviors and perhaps, an increase in preventative strategies and drug policy initiatives in some minority groups when compared to Caucasians. Nevertheless, it is important to pay close attention to race/ethnicity as a possible risk factor for readmission among this population group.

\section{ART adherence}

The non-adherence to ART, when compared to adherence to ART, resulted in a higher likelihood of a 30-day 
readmission rate $(\mathrm{p}<0.01)$. Partly, this can be explained by the co-occurring burden of depression which can make medication compliance an issue due to an increase in despair, isolation, lack of motivation, and sense of neglect $[7,8]$. Therefore, it is important to ensure that ART adherence is maintained in PLWHIV and severe depression.

\section{Insurance status}

Our result also showed that uninsured patients and patients with government insurance (which is categorized as low-moderate socioeconomic status) were more likely to be readmitted in comparison to patients with private insurance (which is categorized as high socioeconomic status). The increase in readmission rate further reiterates the dire need for better means of access to health care services in lower socioeconomic status, especially since access to these resources would improve the quality of care for underserved population groups and improve medication compliance in PLWHIV and depression [9]. It can also play a major role in decreasing hospitalizations, financial burden, and mortality in this population group [10].

\section{Geographic location}

Similarly, rural groups were more likely to be readmitted within 30 days of discharge in comparison to urban/suburban groups in PLWHIV and severe depression. People living in rural localities report lower life satisfaction related to constricted standards of living.

Partly, this may be due to less robust social support structures, limited or nonexistent access to medical and mental health care, or increased social stigmatization related to HIV status as well as mental health services $[9,10]$. These factors can lead to maladaptive coping patterns, poor adherence to medications, and an increase in hospitalizations [11]. Hence, it important to screen for severe depression in this population group to decrease the readmission rate within 30 days [12, 13].

\section{Conclusions}

Our research study is unique in that it focuses on a specific mental health disorder: severe depression and identifies certain risk factors for 30-day readmission rates in PLWHIV. It is important to assess for risk factors in this population group since improved prognosis in PLWHIV could lessen the severity of medical and mental health burdens.

Risk factors such as age, ethnicity, and access to mental health care in underserved populations, medication adherence, and geographic location can play a pivotal role in improving outcomes like readmission rate within 30 days of discharge, length of stay, etc. Furthermore, vigilance for these risk factors and screening for severe depression in PLWHIV may help to reduce financial burden, hospitalizations, mortality, and morbidity. However, further research is needed - particularly, a prospective model may be better at establishing the cause-effect relationship.

\section{Additional Information}

\section{Disclosures}

Human subjects: Consent was obtained or waived by all participants in this study. N/A issued approval N/A. N/A. Animal subjects: All authors have confirmed that this study did not involve animal subjects or tissue. Conflicts of interest: In compliance with the ICMJE uniform disclosure form, all authors declare the following: Payment/services info: Only the later $\$ 270.00$ payment for the Cureus Preferred Editing project was sponsored by the REACH Program, grant no. 5H79TI081358-02 from SAMHSA. This supporting organization had no further role in the conceptualization of the project's idea, study design; in the collection, analysis and interpretation of data; in the writing of the report; or in the decision to submit the paper for publication. Financial relationships: All authors have declared that they have no financial relationships at present or within the previous three years with any organizations that might have an interest in the submitted work. Other relationships: All authors have declared that there are no other relationships or activities that could appear to have influenced the submitted work.

\section{Acknowledgements}

The publication of this project's manuscript is sponsored by the Recognizing and Eliminating Disparities in Addiction through Culturally-informed Healthcare (REACH) Program, grant no. 5H79TI081358-02 from Substance Abuse and Mental Health Services Administration (SAMHSA). Supporting organizations had no further role in the study design; in the collection, analysis, and interpretation of data; in the writing of the report; or in the decision to submit the paper for publication.

\section{References}

1. Upadhyay S, Stephenson AL, Smith DG: Readmission rates and their impact on hospital financial performance: a study of washington hospitals. Inquiry. 2019, 56:46958019860386. 
2. Berry SA, Fleishman JA, Moore RD, Gebo KA: Thirty-day hospital readmissions for adults with and without HIV infection. HIV Med. 2016, 17:167-77. 10.1111/hiv.12287

3. Choi SK, Boyle E, Cairney J, Collins EJ, Gardner S, Bacon J, Rourke SB: Prevalence, recurrence, and incidence of current depressive symptoms among people living with HIV in Ontario, Canada: results from the Ontario HIV Treatment Network Cohort Study. PLoS One. 2016, 11:e0165816. 10.1371/journal.pone.0165816

4. Nijhawan AE, Kitchell E, Etherton SS, Duarte P, Halm EA, Jain MK: Half of 30-day hospital readmissions among hiv-infected patients are potentially preventable. AIDS Patient Care STDS. 2015, 29:465-73. 10.1089/apc.2015.0096

5. Nolan CP, O'Donnell PJM, Desderius BM, Mzombwe M, McNairy ML, Peck RN, Kingery JR: Depression screening in HIV-positive Tanzanian adults: comparing the PHQ-2, PHQ-9 and WHO-5 questionnaires. Glob Ment Health (Camb). 2018, 5 :e38. 10.1017/gmh.2018.31

6. Heckman TG, Somlai AM, Kalichman SC, Franzoi SL, Kelly JA: Psychosocial differences between urban and rural people living with HIV/AIDS. J Rural Health. 1998, 14:138-45. 10.1111/j.1748-0361.1998.tb00615.x

7. Kinyanda E, Weiss HA, Levin J, et al.: Incidence and persistence of major depressive disorder among people living with HIV in Uganda. AIDS Behav. 2017, 21:1641-54. 10.1007/s10461-016-1575-7

8. Sasaki Y, Kakimoto K, Dube C, et al.: Adherence to antiretroviral therapy (ART) during the early months of treatment in rural Zambia: influence of demographic characteristics and social surroundings of patients. Ann Clin Microbiol Antimicrob. 2012, 11:34. 10.1186/1476-0711-11-34

9. Blankenship KM, Smoyer AB, Bray SJ, Mattocks K: Black-white disparities in HIV/AIDS: the role of drug policy and the corrections system. J Health Care Poor Underserved. 2005, 16:140-56. 10.1353/hpu.2005.0110

10. Mitchell MM, Nguyen TQ, Isenberg SR, Maragh-Bass AC, Keruly J, Knowlton AR: Psychosocial and service use correlates of health-related quality of life among a vulnerable population living with HIV/AIDS. AIDS Behav. 2017, $21: 1580-7.10 .1007 / \mathrm{s} 10461-016-1589-1$

11. Zukoski AP, Thorburn S: Experiences of stigma and discrimination among adults living with HIV in a low HIV-prevalence context: a qualitative analysis. AIDS Patient Care STDS. 2009, 23:267-76. 10.1089/apc.2008.0168

12. Wu SF: Rapid screening of psychological well-being of patients with chronic illness: reliability and validity test on WHO-5 and PHQ-9 scales. Depress Res Treat. 2014, 2014:239490. 10.1155/2014/239490

13. Lo CC, Runnels RC, Cheng TC: Racial/ethnic differences in HIV testing: an application of the health services utilization model. SAGE Open Med. 2018, 6:2050312118783414. 10.1177/2050312118783414 